\title{
An Infrared Image Super-resolution Reconstruction Method Based on Compressive Sensing
}

\author{
Yuxing Mao, Yan Wang, Jintao Zhou, Haiwei Jia \\ State Key Lab of Power Transmission Equipment \& System Security and New Technology, \\ Chongqing University, Chongqing 400044, China
}

Key words: Infrared image, SRR, CS, difference operation, OMP.

\begin{abstract}
Limited by the properties of infrared detector and camera lens, infrared images are often detail missing and indistinct in vision. The spatial resolution needs to be improved to satisfy the requirements of practical application. Based on compressive sensing (CS) theory, this thesis presents a single image super-resolution reconstruction (SRR) method. With synthetically adopting image degradation model, difference operation-based sparse transformation method and orthogonal matching pursuit (OMP) algorithm, the image SRR problem is transformed into a sparse signal reconstruction issue in CS theory. In our work, the sparse transformation matrix is obtained through difference operation to image, and, the measurement matrix is achieved analytically from the imaging principle of infrared camera. Therefore, the time consumption can be decreased compared with the redundant dictionary obtained by sample training such as K-SVD. The experimental results show that our method can achieve favorable performance and good stability with low algorithm complexity.
\end{abstract}

\section{Introduction}

In the field of image application, detail missing limits image visual effect and target recognition. As image resolution is severely limited by the number and size of the detector pixels, the most direct way to improve image resolution lies in optimizing image sensor manufacturing process to reduce the pixel size and increase the number of pixels. However, reducing pixel size might bring in higher image noise and increasing the number of pixels will reduce efficiency of sensor. Meanwhile, the cost of high-resolution imaging equipment has increased dramatically, which hinders their application and promotion ${ }^{[1]}$.

SRR is a hotspot in the fields of image processing theory and engineering application. And it's an effective method to solve problem about image details missing in specific application scenarios. Based on imaging theory and optical lens model, SRR is built by image processing method, effective algorithm, and computer software technology. It can be used to restore or approximate the real scene of high-resolution (HR) images from low-resolution (LR) images effectively ${ }^{[2]}$.

Harris and coworkers first proposed processing single image in spatial domain ${ }^{[3]}$. In 1984, Tsai and Huang proposed another method to improve image resolution in the frequency domain initially ${ }^{[4]}$. With ever-growing breakthrough in field of signal processing, many excellent algorithms have emerged, such as wavelet decomposition, CS theory, etc. CS theory, nominated top ten breakthroughs by American Technology Review in 2007, shows that you can use nonlinear algorithm to reconstruct signal perfectly from discrete signals captured randomly at far less than the Nyquist sampling rate. Now it is widely used in process of image SRR ${ }^{[5-6]}$.

SRR is the process evolving from LR image to HR image. It's a typical ill-posed problem, thus, extra information must be generated. In the light of the sources of the extra information, SRR methods include interpolation, study-based method and reconstruction-based method ${ }^{[7]}$.The typical interpolation methods consist of nearest neighbor, bilinear, bicubic and spline interpolation. It is the simplest, fastest, but the worst method. Now the main direction of interpolation is to develop edge enhancement algorithm based on edge and texture features and distribution characteristics of image in spatial and transform domain ${ }^{[8-12]}$. The study-based method is to build a symbiotic model for the high and low resolution images pair by sample learning, then the high frequency details are generated and supplemented to LR images ${ }^{[13-16]}$. But the learning process will be complex and 
time-consuming, and sample training method is not conducive to depict image character. The reconstruction-based method can obtain efficacious extra information from LR image sequences and achieve excellent reconstruction performance. The main effective algorithms include frequency domain method $^{[4]}$ and spatial domain method ${ }^{[17-19]}$.

The starting point of this paper is that: the LR image is regarded as a down-sampling of a HR image, and then the HR image is reconstructed using the sparse signal reconstruction algorithm of CS. To accelerate computing speed, the sparse transformation of HR image is obtained directly by the spatial distribution characteristics of image instead of training redundant dictionary. This method can eliminate the dependence on samples and achieve good reconstruction results with fast speed.

\section{Image super-resolution reconstruction method based on difference operation}

\subsection{The principle of image super-resolution reconstruction based on compressive sensing}

Conventional signal compression process is that: collect signals first according to Nyquist sampling theorem ${ }^{[20]}$, and then compress signals. However, collection and compression are made together in CS theory. CS theory mainly includes three aspects: signal sparse representation, construction of signal measurement matrix and signal reconstruction algorithm.

The premise of using CS theory is that signals can be represented sparsely. Natural signals are generally non sparse, but most of them are sparse in some transform domain. A real signal of length $\mathrm{N}$ can be expressed as a column vector $\mathrm{x}$, and two-dimensional image signals can be arranged in one-dimensional signal by row. Suppose $\quad\left\{\psi_{i}\right\}_{i=1}^{N}$ is a set of orthogonal basis in $R^{N}$ space, expressed as matrix: $\Psi=\left[\psi_{1}, \psi_{2}, \ldots \psi_{N}\right]$, then $\mathrm{N}$-dimensional signal $x$ can be expressed as Eq.(1).

$$
x=\sum_{i=1}^{N} \beta_{i} \psi_{i} \quad \text { or } \quad x=\Psi \beta .
$$

where, $\beta \in R^{N \times 1}$ is the projection of $x$ in space $\Psi$. If there is only $\mathrm{K}(\mathrm{K}<<\mathrm{N})$ non-zero coefficients in $\beta$, and the other coefficients are all 0 (or close to 0 ), then we regard signal $x$ as sparse or compressible and $\Psi$ as the sparse matrix of signal $\mathrm{x}$.

According to the Restricted Isometry Property(RIP) ${ }^{[21]}$, we can use an observation matrix $\Phi \in R^{M \times N}(\mathrm{M}<<\mathrm{N})$ (irrelevant to the sparse matrix $\left.\Psi\right)$ to make linear observation on $\mathrm{x}$ to obtain the low dimensional observation $\mathrm{y}$ under the condition that $\mathrm{x}$ can be represented sparsely, then $\mathrm{x}$ can be reconstructed accurately from y by solving the optimization problems. Image dimension reduction takes place in down-sample step according to the imaging process of detector, so the down-sample matrix D can be regard as observation matrix, see Eq.(2).

$$
y=D x .
$$

where, $y \in R^{M \times 1}$ is observation signals, $\quad D \in R^{M \times N}(\mathrm{M}<<\mathrm{N})$ is observation matrix. With matrix $\mathrm{D}$, we can reduce the dimension of $x$ from $N$ to $M$. Since signal $x$ can be sparse, the linear measurement process can be expressed as Eq.(3).

$$
y=D x=D \Psi \beta=A \beta .
$$

where, $\mathrm{A}$ is called sensing matrix. When A meets the RIP criterion, the process of solving $\beta$ is regard as signal reconstruction. Namely, solving the following formula Eq.(4).

$$
\widehat{\beta}=\arg \min \|\beta\|_{l} \quad \text { s.t } \quad y=\mathrm{D} \Psi \beta \text {. }
$$

When $l$ is 0 , it is the optimization problem based on $l_{0}$-norm. $l_{0}$-norm problem is a NP-hard problem and difficult to be solved directly. Theoretical studies showed that the optimization problem of $l_{1}$-norm is equivalent to $l_{0}$-norm. Many methods can be used to solve $l_{1}$-norm problem: greedy algorithm, relaxation algorithm, Bayesian statistical optimization algorithm, etc. Greedy 
algorithm includes Matching Pursuit (MP) and Orthogonal Matching Pursuit (OMP). Experiments show that OMP algorithm can reconstruct signal with high probability and has a faster convergence rate than MP, so we use OMP algorithm to reconstruct signal in this paper.

\subsection{Application of difference operation to achieve SRR of image block}

We also use CS algorithm to reconstruct image in this paper, but select new observation matrix and sparse decomposition matrix which are different from the conventional CS reconstruction algorithm.

Regular observation matrices include random observation matrix (such as Gauss random observation matrix, etc.) and deterministic observation matrix (such as Chirps sensing matrix, etc.). The uncertainty of the random observation matrix needs to be eliminated by multi-average, which is difficult to achieve in hardware circuit. The exact reconstruction using deterministic matrix needs more measurement times. According to detector imaging principle, LR images can be considered as the result of dimension reduction, namely each point in LR image can be regard as the average of adjacent four points in HR image. With a view to practical application, we get down-sampling matrix $\mathrm{D}$ directly using above principle and observations of $\mathrm{D}$ on any image can store main information of original image.

Sparse decomposition is usually achieved by training appropriate dictionaries to represent images, such as Fourier dictionary, K-SVD study-based method etc. These algorithms have good sparse effect on specific images, but they are complex and with long training time. This paper starts from the characteristics that the pixels within a local area of a natural image changes relatively flat, so the difference between a point and the average of its neighboring eight points is close to 0 . Therefore, we can achieve sparse transformation by difference operation. Because the sparse transformation matrix only depends on the size of image and doesn't need training process, the parse transformation method is simple and time-saving.

The theoretical analysis and experimental results show that the correlation between the observation matrix and sparse transformation matrix is low, namely they satisfy the RIP condition well, so image can be reconstructed more accurately.

When the image size is large, it is awful to calculate the transformation matrix. First of all, we divide the LR image into blocks. Then, the HR blocks are reconstructed from the LR image blocks. Finally, all the HR blocks are put together into a complete image. The algorithm flow chart is presented as Fig.1.

\subsubsection{Construction of observation matrix}

With a view to practical application, we get down-sampling matrix D directly based on the idea of "average over four points". The construction process of D is:

1)The pixel at row $i$ column $j$ of block $Y$ is denoted as $Y_{i, j}, i=1,2, \ldots, m, j=1,2, \ldots, n$. The pixel at row $i_{1}$ column $j_{1}$ of block $X$ is expressed as $X_{i_{1}, j_{1}}, i_{1}=1,2, \ldots, 2 m, j_{1}=1,2, \ldots, 2 n$. According to the sampling model, the Eq.(5) are obtained.

$$
\mathrm{Y}_{\mathrm{i}, \mathrm{j}}=\left(\mathrm{X}_{2(\mathrm{i}-1)+1,2(\mathrm{j}-1)+1}+\mathrm{X}_{2(\mathrm{i}-1)+2,2(\mathrm{j}-1)+1}+\mathrm{X}_{2(\mathrm{i}-1)+1,2(\mathrm{j}-1)+2}+\mathrm{X}_{2(\mathrm{i}-1)+2,2(\mathrm{j}-1)+2}\right) / 4 .
$$

2)According to the construction method of column vector, $Y_{i, j}$ is the $n(i-1)+j$ element of column vector $y$, expressed as $y_{\mathrm{n}(\mathrm{i}-1)+\mathrm{j}}$. Similarly, $X_{i_{1}, j_{1}}$ is expressed as $x_{2 \mathrm{n}\left(\mathrm{i}_{1}-1\right)+\mathrm{j}_{1}}$. So Eq.(5) can be rewritten as Eq.(6).

$$
\mathrm{y}_{\mathrm{n}(\mathrm{i}-1)+\mathrm{j}}=\left(\mathrm{x}_{2 \mathrm{n}(2(\mathrm{i}-1))+2(\mathrm{j}-1)+1}+\mathrm{x}_{2 \mathrm{n}(2(\mathrm{i}-1))+2(\mathrm{j}-1)+2}+\mathrm{x}_{2 \mathrm{n}(2(\mathrm{i}-1)+1)+2(\mathrm{j}-1)+1}+\mathrm{x}_{2 \mathrm{n}(2(\mathrm{i}-1)+1)+2(\mathrm{j}-1)+2}\right) / 4 \text {. }
$$

3)For Eq.(6), changing $i$ from 1 to $m$ and $j$ from 1 to $n$, we can get $m \times n$ calculation formulas corresponding to $y_{1} 、 y_{2} \ldots y_{\mathrm{mn}}$, which reflect that each element of vector $\mathrm{Y}$ is related to some four elements of vector $x$. The down-sampling matrix $D$ with $m=n=4$ is presented as Fig. 2. 


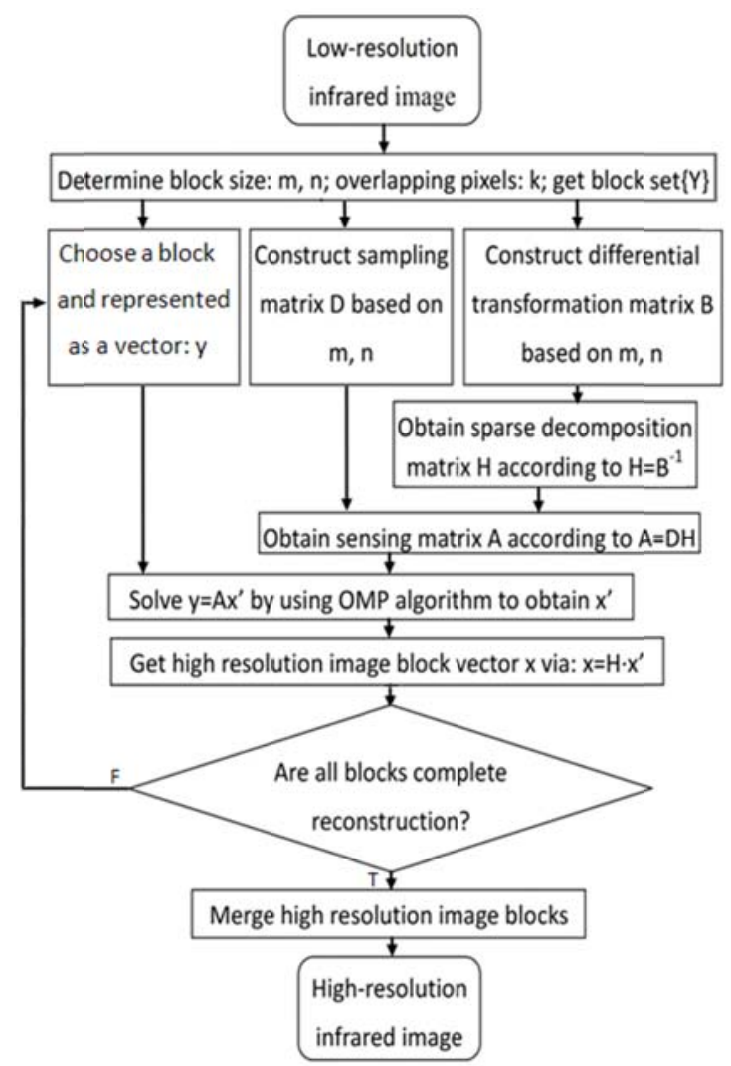

Fig. 1 algorithm flow chart of this paper

\begin{tabular}{|c|c|c|c|c|c|c|c|c|c|c|c|c|c|c|c|c|c|c|c|c|c|}
\hline \multirow[b]{2}{*}{ Row 1} & \multicolumn{7}{|c|}{ Columns $1-8$} & \multicolumn{6}{|c|}{ Columns 9-16 } & \multicolumn{8}{|c|}{ Columns 17-64 } \\
\hline & 0.25 & 0.25 & 0 & 0 & 0 & $\cdots$ & 0 & 0.25 & 0.25 & 0 & 0 & 0 & $\cdots$ & 0 & 0 & $\cdots$ & 0 & 0 & 0 & 0 & 0 \\
\hline Row2 & 0 & 0 & 0.25 & 0.25 & 0 & $\cdots$ & 0 & 0 & 0 & 0.25 & 0.25 & 0 & $\cdots$ & 0 & 0 & $\cdots$ & 0 & 0 & 0 & 0 & 0 \\
\hline & & & & $\vdots$ & & & & & & & $\vdots$ & & & & & & & $\vdots$ & & & \\
\hline Row 16 & 0 & 0 & 0 & 0 & 0 & $\cdots$ & 0 & 0 & $\cdots$ & 0 & 0 & 0 & 0.25 & 0.25 & 0 & $\cdots$ & 0 & 0 & 0 & 0.25 & 0.25 \\
\hline
\end{tabular}

Fig. 2 the down-sampling matrix $D$ with $m=n=4$

\subsubsection{Construction of sparse transformation matrix}

As the difference between one pixel and the average of its eight neighboring pixels is close to 0 , we can obtain sparse transformation matrix directly.

In 2.2.1, $X$ has been defined as a HR image block and $X_{i_{1}, j_{1}}$ is the pixel value of the pixel at row $i_{1}$ column $j_{1}$. Here, we define $X^{\prime}$ as the transform block of $X$ and $X_{i_{1}, j_{1}}^{\prime}$ is the pixel value of the pixel at row $i_{1}$ column $j_{1}$. The construction steps of sparse transformation matrix are as following:

1)The pixel values of non boundary points of $X^{\prime}$ are obtained by the difference operation between the corresponding points and its neighborhood points of the original block $X$, represented by Eq.(7).

$$
X_{i_{1}, j_{1}}^{\prime}=X_{i_{1}, j_{1}}-\left(X_{i_{1}-1, j_{1}-1}+X_{i_{1}-1, j_{1}}+X_{i_{1}-1, j_{1}+1}+X_{i_{1}, j_{1}-1}+X_{i_{1}, j_{1}+1}+X_{i_{1}+1, j_{1}-1} X_{i_{1}+1, j_{1}} X_{i_{1}+1, j_{1}+1}\right) / 8
$$

2)Processing method of the boundary points. We use different extension methods to ensure the difference transformation matrix is full rank (reversible): use mirroring extension to deal with the points on first row and first column, and zero extension to the points on last line and last column.

3)The transform block $X^{\prime}$ is arranged into a column vector $x^{\prime}$ according to principle "row first and 
column after", namely $X_{i_{1}, j_{1}}^{\prime}$ into $x_{2 \mathrm{n}\left(\mathrm{i}_{1}-1\right)+\mathrm{j}_{1}}^{\prime}$. The Eq.(7) can be written in Eq.(8).

$$
\begin{gathered}
x_{2 \mathrm{n}\left(\mathrm{i}_{1}-1\right)+\mathrm{j}_{1}}^{\prime}=x_{2 \mathrm{n}\left(\mathrm{i}_{1}-1\right)+\mathrm{j}_{1}}-\left(x_{2 \mathrm{n}\left(\mathrm{i}_{1}-2\right)+\mathrm{j}_{1}-1}+x_{2 \mathrm{n}\left(\mathrm{i}_{1}-2\right)+\mathrm{j}_{1}}+x_{2 \mathrm{n}\left(\mathrm{i}_{1}-2\right)+\mathrm{j}_{1}+1}+\right. \\
\left.x_{2 \mathrm{n}\left(\mathrm{i}_{1}-1\right)+\mathrm{j}_{1}-1}+x_{2 \mathrm{n}\left(\mathrm{i}_{1}-1\right)+\mathrm{j}_{1}+1}+x_{2 \mathrm{ni}_{1}+\mathrm{j}_{1}-1}+x_{2 \mathrm{ni}_{1}+\mathrm{j}_{1}}+x_{2 \mathrm{ni}_{1}+\mathrm{j}_{1}+1}\right) / 8
\end{gathered} .
$$

4)For Eq.(8), changing $i_{1}$ from 1 to $2 \mathrm{~m}$ and $j_{1}$ from 1 to $2 \mathrm{n}$, we can get $2 m \times 2 n$ calculation formulas corresponding to $x_{1}^{\prime} 、 x_{2}^{\prime} \ldots x_{4 \mathrm{mn}}^{\prime}$, which reflect each element of vector $x^{\prime}$ is related to some nine elements of vector $x$ (except boundary points). The formulas of all elements of vector $x^{\prime}$ are written in matrix form as $x^{\prime}=B x$, then we substitute $H=B^{-1}$ into it and obtain Eq.(9).

$x=H x^{\prime}$.

Where, $H$ is the sparse transformation matrix in this paper and $x^{\prime}$ is sparse. Difference transformation matrix $\mathrm{B}$ with $\mathrm{m}=\mathrm{n}=4$ is presented as Fig.3.

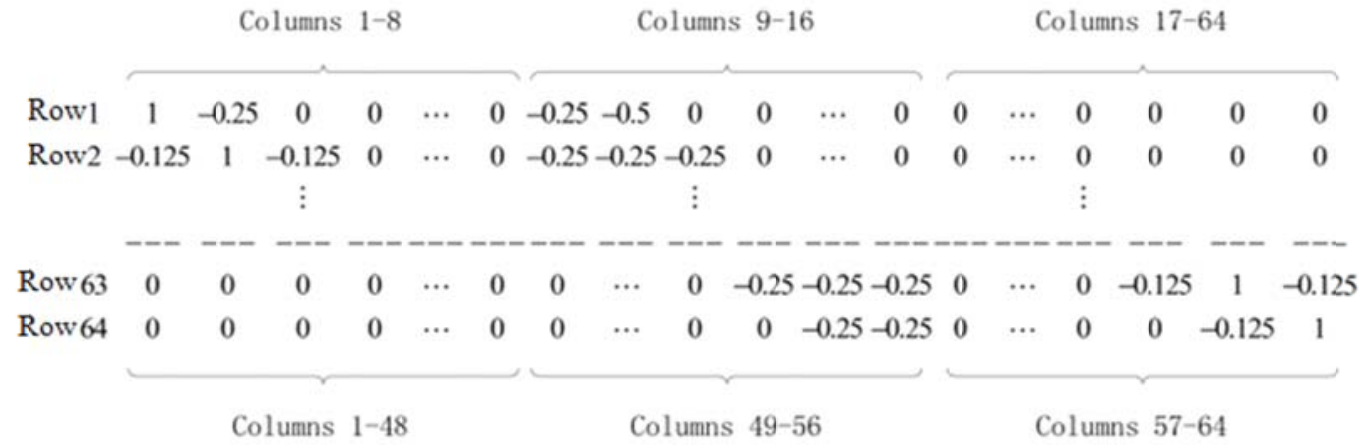

Fig. 3 difference transformation matrix $B$ with $m=n=4$

\subsubsection{Reconstruction of HR image block}

After the problems of observation and sparse decomposition solved above, the sparse signal can be reconstructed with high probability from a few random observations. The reconstruction steps are as following:

1)Eq.(9) is substitute to Eq.(2), then, Eq.(10) can be obtained.

$$
y=D H x^{\prime}=A x^{\prime} .
$$

Where, the dimension of the sensing matrix $\mathrm{A}$ is $m n \times 4 m n$. There are many zero values in $x^{\prime}$, so it can meet the reconstruction condition of CS theory.

2)In Eq.(10), y is the observation vector of LR image blocks, and the sensing matrix A can be obtained by $A=D H$, then we can use OMP algorithm to reconstruct the sparse vector $x^{\prime}$.

3)Substitute $x^{\prime}$ in Eq.(9) to calculate vector $\mathrm{x}$, and then rearrange $\mathrm{x}$ into matrix form of $2 \mathrm{~m} \times 2 \mathrm{n}$ to get the HR image block $X$.

After all HR image blocks are obtained, they are merged to a complete HR image.

\section{Experimental result analysis}

Three LR infrared images were collected for testing, as shown in Figure 4. The resolution are all $160 \times 120$. With $m=n=4$, the number of blocks in horizontal (vertical) direction of these images is all 40(30). These HR images are reconstructed as $320 \times 240$ by using the following algorithm respectively: bicubic interpolation algorithm, reconstruction algorithm based on DCT dictionary, reconstruction algorithm based on K-SVD dictionary, and the method of this paper. The reconstruction results are shown in Figure 5. 


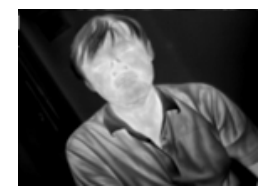

(a)

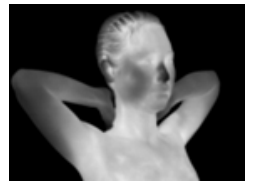

(b)

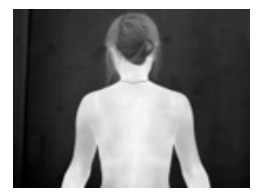

(c)

Fig. 4 LR infrared image used for algorithm testing
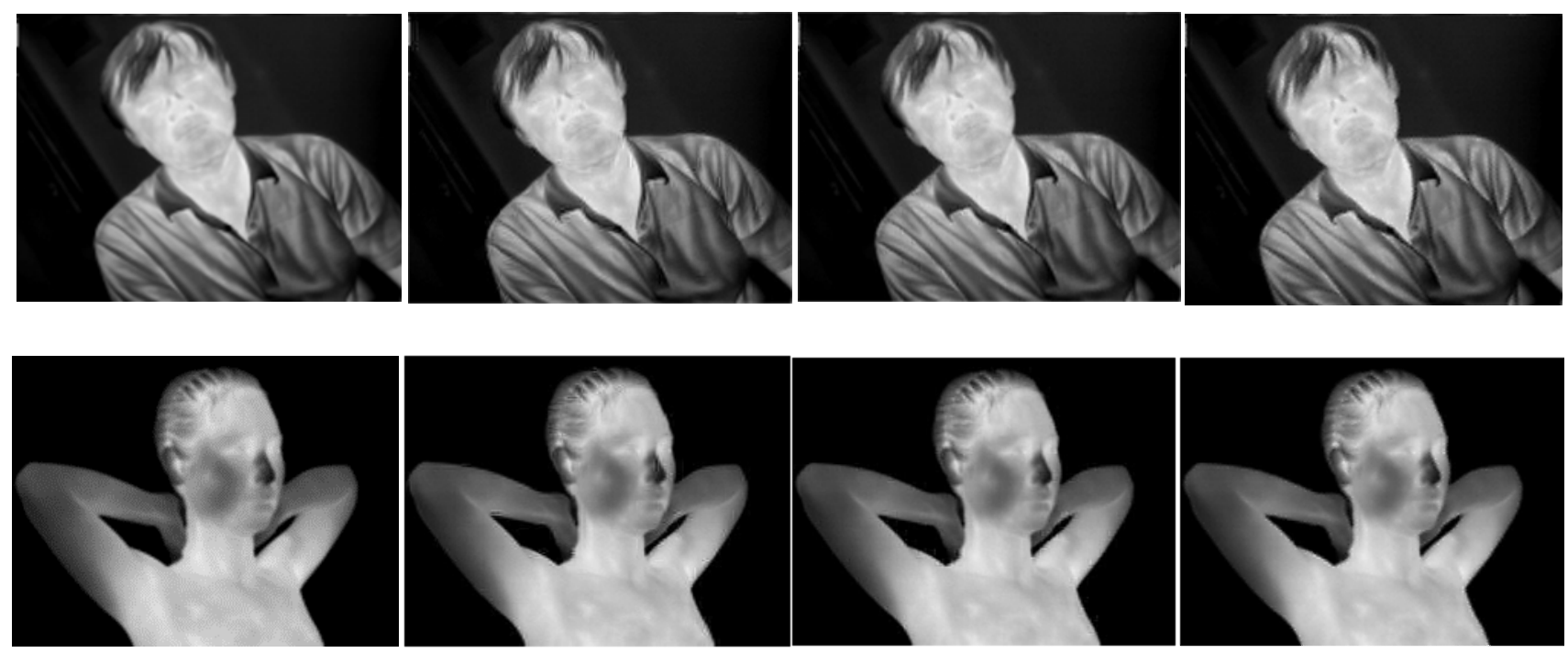

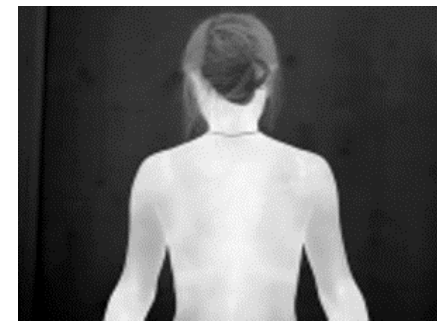

(1)

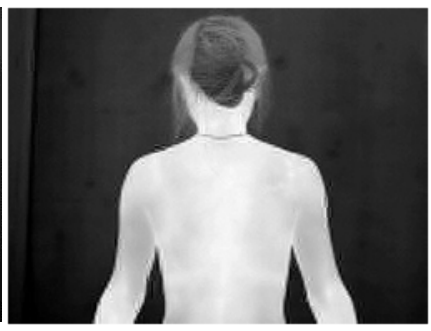

(2)

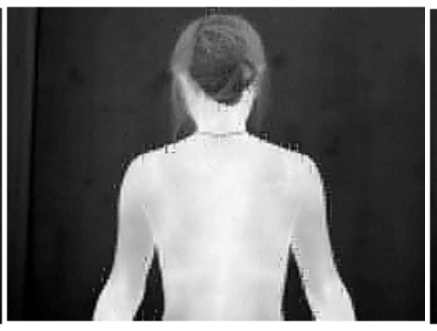

(3)

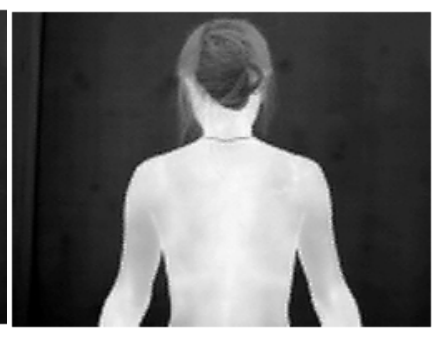

(4)

Fig. 5 Comparison of the four algorithms: (1) Bicubic interpolation. (2) Reconstruction based on DCT. (3) Reconstruction based on K-SVD. (4) Algorithm of the paper

It can be seen from Fig. 5 that: The reconstruction images of bicubic interpolation are fuzzy and details unclear. The algorithm based on DCT dictionary has a good effect on the reconstruction of (a), but the reconstructed images of image (b) and (c) have ripples on the edge. The algorithm based on K-SVD dictionary is also good for the image (a), but there are some glitches on the edges with (b) and (c). However, the algorithm proposed in this paper showed good visual effect on all the reconstruction image (a), (b), (c). In addition, the time consumption of the four algorithms are compared. The reconstruction time of the four algorithms of the three LR images are shown in Table 1:

Table 1 reconstruction time of four algorithms

\begin{tabular}{ccccc}
\hline $\begin{array}{c}\text { image } \\
\text { algorithms }\end{array}$ & $\begin{array}{c}\text { bicubic } \\
\text { interpolation }\end{array}$ & based on DCT & based on K-SVD & the paper's \\
\hline image(a) & $0.079941[\mathrm{~S}]$ & $28.442470[\mathrm{~S}]$ & $527.143008[\mathrm{~S}]$ & $27.156478[\mathrm{~S}]$ \\
image(b) & $0.079603[\mathrm{~S}]$ & $17.738835[\mathrm{~S}]$ & $506.582265[\mathrm{~S}]$ & $16.174587[\mathrm{~S}]$ \\
image(c) & $0.144095[\mathrm{~S}]$ & $28.209859[\mathrm{~S}]$ & $607.361172[\mathrm{~S}]$ & $27.675344[\mathrm{~S}]$ \\
\hline
\end{tabular}

It is clear from the table that the bicubic interpolation algorithm exhibits absolute advantages in time cost, but the reconstruction images are all indistinct in vision. The algorithm based on K-SVD needs the longest time due to training dictionary, so it is difficult to be used in system with high 
real-time requirements. We adopt fixed DCT dictionary(without training process) in the algorithm based on DCT dictionary, thus, the algorithm is more time-saving than K-SVD, but the visual effect of reconstruction is varied within different pictures, and this hinders its further popularization. After comprehensive comparison, the proposed algorithm is time-saving, with satisfactory results, no bias for images, strong adaptability, and also good robustness.

\section{Conclusion}

A single infrared image SRR algorithm is proposed in this paper, which synthetically adopts image sampling model, image difference transformation algorithm and CS theory. Advantages of this algorithm include: it's easy to achieve just using a single image; transform matrix can be constructed directly via difference operation without training process; sparse transformation based on difference operation is simple, fast and stable; thus it is especially suitable for infrared image SRR.

Since reconstruction results may produce white noise when complex image details are involved, further improvement of the algorithm is needed. This phenomenon can be explained as: there are some correlation between observation matrix D and sparse matrix B, which may introduce some errors in image reconstruction and even cause the reconstruction of certain points to fail. In this paper, matrix D and matrix B are constructed separately, namely the irrelevant constraint conditions are not integrated into the construction of sparse matrix. But further research should be taken to construct a sparse matrix with lower correlation to the observation matrix under the RIP constraint.

\section{Acknowledgment}

This work is supported by the project of National Natural Science Foundation of China (61571068) and the innovative research projects of colleges and universities in Chongqing (12A19369).

\section{References}

[1] Zhang Liang-pei, Shen Huan-feng, Zhang Hong-yan, Yuan Qiang-qiang. Super resolution image reconstruction [M]. First edition. Beijing: Science Press, 2012.8

[2] Kang M, Chaudhuris 2003 IEEE Signal Proc. Mag. 201920

[3] Rui Song. Research on Super Resolution Reconstruction of Video and Image Sequences [D]. Xi'an: XiDian University, 2009

[4] Tsai R Y, Huang T S. Multiframe image restoration and registration. in Advances in Computer Vision and Image Processingar JAI Press Inc.,1984, 317-339.

[5] Jiao PF, Li L, Zhao J. New advances of compressed sensing in medical image reconstruction[J]. CT Theory and Applications, 2012, 21(1): 133-147.

[6] REN Yue-Mei ${ }^{[1]}$, ZHANG Yan-Ning ${ }^{[2]}$, LI Ying ${ }^{[2]}$. Advances and Perspective on Compressed Sensing and Application on Image Processing [J]. Acta Automatica Sinica, 2014, 40(8): 1563-1575.

[7] JIANG Jing, ZHANG Xue-song. A Review of Super-resolution Reconstruction Algorithms [J]. Infrared Technology, 2012,01:24-30.

[8] Chang S G, Cvetkovi Z, Vetterli M. Locally adaptive wavelet-based image interpolation [J]. IEEE Transactions on Image Processing, 2006, 15(6):1471-1485.

[9] Cheng Guang-quan, Cheng Li-zhi. Direction Adaptive Image Interpolation via Wavelet Transform [J]. JOURNAL OF ELECTRONICS \& INFORMATION TECHNOLOGY, 2009, 31(2): 265-269

[10]ZHAO Liang, HE Hong-jie, YIN Zhong-ke. Texture direction adaptive image interpolation based on Curvelet transform [J]. Journal of Optoelectronics.Laser, 2012,04: 798-804.

[11]J. J. Wang, Y. H. Gong. Fast image super resolution using connected component enhancement[C]. Proceedings of IEEE International Conference on Multimedia and Expo(ICME), Hannover, German, June 2008, 157-160 
[12]Qianying Zhang, JitaoWu. Image super- resolution using windowed ordinary Kriging interpolation [J]. Optics Communications, 2015, 336(2015)140-145.

[13]Zongxu Pan, Jing Yu, Huijuan Huang, Shaoxing Hu, Aiwu Zhang, Hongbing Ma, and Weidong Sun. Super-Resolution Based on Compressive Sensing and Structural Self-Similarity for Remote Sensing Images[J]. IEEE TRANSACTIONS ON GEOSCIENCE AND REMOTE SENSING, 2013, 51(9):4864-4876.

[14]Dong Sun, Qingwei Gao, Yixiang Lu, Lijun Zheng, Hui Wang. A high quality single-image super-resolution algorithm based on linear Bayesian MAP estimation with sparsity prior[J]. Digital Signal Processing, 2015, 35 (2014): 45-52.

[15]Deng Cheng-Zhi ${ }^{[1]}$, Tian Wei ${ }^{[1]}$, Chen Pan ${ }^{[2]}$, Wang Sheng-Qian ${ }^{[1]}$, Zhu Hua-Sheng ${ }^{[1]}$, Hu Sai-Feng ${ }^{[1]}$. Infrared image super-resolution via to cality-constrained group sparse model [J]. Acta Physica Sinica, 2014, 63(4): 044202-1 044202-8.

[16]Xiaoyan Li, Hongjie He , Zhongke Yin, FanChen, JunCheng. KPLS-based image super-resolution using clustering and weighted boosting [J]. Neurocomputing, 2015, 149(2015)940-948.

[17]LI Zhan ${ }^{[1]}$, ZHANG Qing-Feng ${ }^{[1]}$, MENG Xiao-Hua ${ }^{[1]}$, LIANG Peng ${ }^{[2]}$, LIU Yu-Bao ${ }^{[3]}$. Super-resolution Reconstruction for Multi-resolution Image Sequence [J]. Acta Automatica Sinica, 2012, 38(11): 1804-1814.

[18]Xu Feng ${ }^{[1]}$, Yan Xijun ${ }^{[1]}$, Huang Chenrong ${ }^{[2]}$, Zheng Shengnan ${ }^{[2]}$, Huang Fengchen ${ }^{[1]}$, Xu Lizhong $^{[1]}$. Normalized convolution based super-resolution reconstruction using feature-driven prior [J]. Journal of Image and Graphics,2014,19(10): 1514-1523.

[19]Ramy M. Bahy, Gouda I. Salama, Tarek A. Mahmoud. Adaptive regularization-based super resolution reconstruction technique for multi-focus low-resolution images[J]. SIGNAL PROCESSING, 2014,103():155-167.

[20]Farsiu S, Robinson M D, Elad M, Milanfar P. Fast and robust multiframe super resolution. IEEE Transactions on Image Processing, 2004, 13(10): 1327-1344.

[21]Candes E, Romberg J. Sparsity and incoherence in compressive sampling[J]. Inverse Problems, 2007, 23(3):969-985. 